\title{
Meson2006 Summary: Theory
}

\author{
T.Barnes \\ Physics Division, Oak Ridge National Laboratory, Oak Ridge, TN 37831, USA \\ Department of Physics and Astronomy, University of Tennessee, Knoxville, TN 37996, USA.
}

\begin{abstract}
This is a summary of theoretical plenary contributions to the biennial hadron physics conference Meson2006, which was the ninth in this series. The topics covered in the meeting include low energy pion-pion and pion-nucleon interactions, photoproduction and hadronic production of light mesons and baryons, in-medium effects, recent developments in charmed mesons, charmonia and B mesons, the status of exotica, and some related topics such as final state interactions. In this contribution we review and summarize the plenary talks presented by theorists at the meeting, and emphasize some of the main points of their presentations. Where appropriate we will add brief comments on some aspects of QCD spectroscopy. Finally, following tradition, we conclude with a new Feynman story.

PACS numbers: 14.20.-c, 14.40.-n
\end{abstract}

\section{INTRODUCTION}

The biennial Meson conference series has historically been hosted by a long-standing collaboration between the German and Polish nuclear and hadron physics communities, especially involving research institutions at Kraków and Jülich. The range of topics represented at this meeting since the inception in 1991 has grown to span much of the physics of QCD spectroscopy. As a result of the history of this meeting, there was considerable coverage of the physics of mesons below $1 \mathrm{GeV}$, strange mesons, light $u, d, s$ baryon spectroscopy, and in-medium effects. The recent activity in other areas of QCD, such as the QGP searches at RHIC and the new discoveries in charm spectroscopy, have led to new research areas in hadron physics that are now also major components of this meeting.

Here we summarize the plenary theory contributions at Meson2006, as well as some especially theory-relevant experimental presentations, ordered according to the broad topics discussed above: light hadrons, medium effects, heavy quark hadron spectroscopy and multiquark physics. Of course there is considerable overlap between these areas in some contributions, which I will stress where appropriate.

\section{A. Plenary contributions on light hadrons}

\section{Prof. Leutwyler and the Red Dragon}

We begin our summary with the presentation on the lightest of hadrons. H.Leutwyler [1, 2] discussed the status of the $\sigma$ meson (the "Rote Drache") in $\pi \pi$ scattering. Leutwyler noted that the Roy equations, combined with dispersion relations, give an accurate numerical description of low-energy $\pi \pi$ scattering in terms of just three numbers, which he takes to be the scattering lengths $a_{0}$, $a_{2}$ and a phase shift $\delta$. In this talk Leutwyler reported the interesting new result that the location of scattering amplitude poles on the $2^{\text {nd }}$ sheet is accurately determined by the (more accessible) location of zeros on the $1^{\text {st }}$ sheet. This new approach allows a much more accurate determination of the location of the $\sigma$ pole, with the result

$$
\text { Pole position }=441(4)-272(6) i \mathrm{MeV} \text {. }
$$

Since there are twin poles in this approach with equal magnitude \pm imaginary parts, and they are at the lowenergy "head" of the $\mathrm{I}=0 \pi \pi$ scattering amplitude in the complex E plane, it is amusing to refer to these poles as the eyes of the red dragon. Thus, what Prof. Leutwyler has discovered can be summarized in Latin, as appropriate for a conference in a famous medieval city, as the

\section{"Loci Oculorum Draconis Rutili".}

Although the location of the eyes of the dragon is now well established, the actual nature of the associated $\sigma$ meson state is unfortunately not specified by this method. Thus we now know where the $\sigma$ is, but not what it is.

\section{Kd scattering}

Since the $K^{-} p$ and $K^{-} n$ scattering amplitudes near threshold are at least moderately well-known experimentally, one may use this information to evaluate the $K^{-} d$ scattering length. This theoretical study was reported by A.Gal [3, 4], and is an improvement on previous work in not using a fixed-center approximation. Their initial conclusion is that the (complex) $K^{-} d$ scattering length is fairly large, $\sim 1-2 \mathrm{fm}$. Based on this work, they quote an ultimate goal for the accuracy of this scattering length of $\approx 10 \%$ for theory, and (at the DEAR/SIDDHARTA experiment at $\mathrm{DA} \Phi \mathrm{NE}) \approx 5 \%$ for experiment.

\section{Isospin violation in the strong interaction}

In this contribution, Niskanen discussed prospects for observing evidence for isospin violation in the strong interaction in the processes $N N \rightarrow d \pi[\underline{5},[\underline{6}]$. (We use $N$ 
generically to represent a nucleon, and $p$ and $n$ specifically for protons and neutrons.) Of course relatively weak isospin violation is expected in the strong interaction from several sources, such as electromagnetic corrections, the $u, d$ quark mass differences, and (presumably indirectly due to these effects) the $p, n$ and other hadron isomultiplet mass differences. In the naive isospin limit one has

$$
\frac{p p \rightarrow d \pi^{+}}{p n \rightarrow d \pi^{0}}=2
$$

Although one can search for violations of this ratio, there are various complications such as the choice of the kinematic point at which the cross sections are to be compared, and the difference in quality of $p$ and $n$ beams. After a review of these complications and a long and lively discussion with the audience, Niskanen offered a new uncertainty principle, which relates the ease $E$ of the experiment and the associated theoretical interpretation:

$$
E_{\text {expt. }} \cdot E_{\text {theor. }} \geq \text { constant. }
$$

\section{Baryon resonance photoproduction, $\gamma N \rightarrow \eta N$ and $\eta^{\prime} N$.}

Although this was an experimental contribution, there were sufficiently interesting theoretical issues discussed to merit a mention in this theory summary. In this talk Tiator [7] discussed the photoproduction of $\eta N$ and $\eta^{\prime} N$ final states, using the Mainz isobar analysis program [8] for pseudoscalar photoproduction. This reaction was assumed to be dominated by two Feynman diagrams, $t$ channel vector meson exchange ( $\rho$ and $\omega$ are included) and $s$-channel nucleon and $N^{*}$ resonance production. Two interesting and rather unsettling observations made by Tiator were that

1) the $D_{15}(1675) \rightarrow \eta N$ branching fraction in the fit varies from $0.7 \%$ to $17 \%$, depending on the assumed $t$ dependence of vector exchange (Regge versus form factors), and

2) the best fit gives a tiny $N N \eta$ coupling, with $g_{N N \eta}^{2} / 4 \pi$ less that an order of magnitude smaller than would be expected from $\mathrm{SU}(3)$ symmetry.

Since the quark model (see Table I of Downum et al. [11]) and the Nijmegen $N N$ force model [12] both anticipate that $g_{N N \eta}$ should be comparable to $g_{N N \pi}$, which involves a simple $\mathrm{SU}(3)$ flavor factor, this suggests that the experimental analysis may not be giving realistic couplings. Whatever the explanation, this is certainly a striking result.

\section{Baryon resonance photoproduction, $\gamma N \rightarrow \phi \eta N$.}

Soyeur [9, 10] discussed a very interesting baryon resonance photoproduction process, $\gamma N \rightarrow \phi \eta N$. In her theoretical analysis she assumed that this reaction at low energies is dominated by $t$-channel $\pi$ and $\eta$ meson exchange, with the $\phi$ meson produced through $\gamma \pi \phi$ and $\gamma \eta \phi$ vertices. (These of course have known couplings.) The final $\eta$ is assumed to be produced by an intermediate $N^{*}(1535)$. This is a nice example of a coupledchannel process, since it is sensitive to the interference of the off-diagonal amplitude for $\pi N \rightarrow N^{*}(1535) \rightarrow \eta N$ and the diagonal amplitude for $\eta N \rightarrow N^{*}(1535) \rightarrow \eta N$. An experimental study of this reaction can also establish the relative phase of the $\pi N$ and $\eta N$ couplings of the $N^{*}(1535)$, which is predicted by the quark model. Soyeur suggests that this would be an interesting reaction to study at JLAB, with $\mathrm{E}_{\gamma} \approx 4 \mathrm{GeV}$.

\section{B. Plenary contributions on in-medium effects}

There were several experimental contributions on inmedium effects on hadron masses and widths, especially on vector mesons, by Weygand, Pietraszko, Yokkaichi and Krusche. Although this is certainly an interesting and active topic, one had an uncomfortable initial feeling during these presentations that the theoretical underpinning of this field was not strong. Each experimental talk made reference to the same iconic figure of the $\langle\bar{q} q\rangle$ condensate versus temperature and density, and stated that this somehow explained the fall in meson mass with increasing density, without a critical discussion of the physics issues. Even if this picture is correct, experiments do not measure a condensate; they measure a cross section, a resonance mass and width, or some other set of observables. The interpretation of mass shifts in terms of a $\langle\bar{q} q\rangle$ condensate may be misleading or even incorrect, and is certainly not what experiments on in-medium hadron properties themselves contribute.

In view of this rather uncritical experimental support for a theoretical concept, the subsequent theoretical review of in-medium effects by Mosel [13] was refreshing indeed. Mosel noted that the in-medium hadron mass shifts are not magic, instead they are (mostly) simple FSI effects [14]. Mosel et al. have developed codes to simulate these FSI effects, and find that several of the well known medium modifications can easily be explained in this manner. One example is the well-established doubling of the $\Delta$ width, which they find is due to $\Delta N$ final state interactions $(\Delta N \rightarrow N N$ and $\Delta N N \rightarrow N N N)$. Another is the downwards mass shift of the $\pi \pi$ system; "A big part of the $\pi \pi$ mass shift is due to $\pi N$ rescattering on the way out." Finally, Mosel warns that the relation between these mass shifts and $\langle\bar{q} q\rangle$ is obscure at best: "The connection of any hadron mass with the fall-off of $\langle\bar{q} q\rangle$ is very indirect." 


\section{Plenary contributions on heavy-quark hadrons}

The very exciting recent developments in the spectroscopy of charm were also discussed at Meson2006. As these were almost exclusively experimental talks, they are not reviewed here, and will instead be summarized in the experimental review by Seth [15. The specific topics covered were the $\mathrm{X}(1835)$ (C.Zhang; this was actually a presentation on a light hadrons topic), B decays to light mesons (I. Gough Eschrich), $c$ mesons at Belle (S.Korpar) charmonium at HERA (A.Meyer), $c$ and $c \bar{c}$ spectroscopy at BABAR (M.Pelizäus), $\mathrm{D}_{s}$ and $c \bar{c}$ spectroscopy at CLEO (M.Shepherd) and $c$ physics at FOCUS (C.Göbel).

The single theoretical talk in this area was by A.Krassnigg [16], who reviewed developments in a DysonSchwinger approach to meson spectroscopy [17]. In this approach one solves coupled integral equations for selfenergy and vertex functions, given a truncation scheme for $n$-point functions. Their approach has the advantage that it respects chiral symmetry and is relativistic. Thus far it has been applied to ground-state pseudoscalars and vectors, and is now being extended to radial excitations, scalars and axial vectors. This is a "work in progress", for example their current result for the $\eta_{c}^{\prime}$ mass is a rather low $3.45 \mathrm{GeV}$.

\section{Plenary contribution on multiquarks}

As a final physics topic, the experimental presentation by Kabana [18, 19] on the status of pentaquarks raised some familiar questions about multiquark states, which may be worth stressing yet again in this summary. As was noted in the HADRON05 Theory Summary [20], there is a fundamental theoretical problem with light multiquark resonances such as the pentaquark, which despite three decades of work in this area is still not widely appreciated. One is reminded of a quote from a Mel Brooks movie regarding earlier reports of monstrous scientific discoveries;

"These are very serious charges you're making, and all the more painful to us, your elders, because we still have nightmares from five times before."

$$
\text { - village elder, Young Frankenstein 21] }
$$

The problem with multiquarks is that if a multiquark system such as $q^{2} \bar{q}^{2}$ is above the threshold for dissociation into two $q \bar{q}$ mesons (such as a $\mathrm{J}^{P C}=0^{++} u^{2} \bar{d}^{2}$ above $2 m_{\pi}$, or a $\theta(1540) q^{4} \bar{s}$ pentaquark above $K N$ ), this system can simply "fall-apart" into two lighter hadrons without a decay interaction. Although this has entered physics folklore as the notion that multiquark systems will be very broad, the actual effect of fall-apart modes below threshold may be much more drastic; without a fission barrier to suppress dissociation, these multiquark systems need not form resonances at all. This option, the absence of light multiquark resonances due to fallapart modes, was appreciated in some of the theoretical literature on multiquarks in the late 1970s 22], but is in danger of being forgotten. For this reason, Isgur's warning about the recurring "Multiquark Fiasco" [23] should be required reading for anyone working on the physics of multiquarks.

\section{FEYNMAN STORY}

This section continues my "tradition" of ending Meson with a new Feynman story 24]. The story of the pentaquark has shown us yet again the importance of rigorous integrity in science, for example through meticulously listing and checking every conceivable alternative explanation for an apparently positive experimental signal. Feynman refers to this in his famous lecture on "Cargo Cult Science" 25], in which he discusses what can go wrong in scientific efforts that are not careful to maintain an appropriate level of skepticism:

"...there is one feature I notice that is generally missing in cargo cult science..."

"It's a kind of scientific integrity, a principle of scientific thought that corresponds to a kind of utter honesty - a kind of leaning over backwards. For example, if you're doing an experiment, you should report everything that you think might make it invalid - not only what you think is right about it: other causes that could possibly explain your results; and things you thought of that you've eliminated by some other experiment, and how they worked - to make sure the other fellow can tell they have been eliminated."

The theorists' analog of this scientific integrity presumably includes being aware of and acknowledging all relevant experimental results, especially those that may not support the theoretical ideas being developed. This brings me to my Feynman story. More accurately it is an anti-Feynman story, since it ends with him stating the opposite of his principle of scientific ethics for effect.

As a graduate student in Caltech in the mid-1970s, I and several of the other theory students supported ourselves as TAs by grading graduate-level physics classes. One that I graded while it was taught by my advisor Jon Mathews was PHY205, which was an advanced quantum mechanics class. During a two week period in which Mathews was not available he asked Feynman to substitute for him. Of course we graduate students were excited about Feynman teaching the class, although in some ways he was not the most widely appreciated graduate physics instructor at Caltech; his notes were typically rather incomplete, so his in-class derivations would have mistakes that he would correct by fudging signs and changing overall factors, based on what he knew the right answer should be at the end. This made for an interesting lecture, but taking notes was rather difficult. Feynman decided to teach us about the quark model in this class, 
including meson and baryon state vectors and some resulting predictions. In the final stages of these lectures we were told about magnetic moments, which was a famous success of the quark model. Indeed, Feynman stated that he first believed in the quark model when the baryon magnetic moments started coming out right.

This topic was especially exciting for me, since I had been calculating off-diagonal magnetic transition moments among mesons in the quark model, and I knew that there were new experimental results that were in clear disagreement with the quark model predictions. So, as a dutiful TA for the class, I spoke up and noted that there were also interesting transition moments; Feynman agreed with this, and made a few statements about them as well. I then stated that there were new experimental results that disagreed with those predictions. He was evidently not aware of this. After class I gathered up these new experimental papers and went to Feynman's office, thinking that he would be very interested in hearing about these results. I knocked on the door, Feynman opened it, gave me a mock-angry smile, and said

"What's the idea, messing up a perfectly good lecture by referring to experiment?"

\section{Acknowledgments}

I would like to thank the organisers for their kind invitation to give this Meson2006 (theory) summary talk, and for the opportunity to discuss the physics of hadrons with my fellow participants. This research was supported in part by the U.S. National Science Foundation through grant NSF-PHY-0244786 at the University of Tennessee, and the U.S. Department of Energy under contract DEAC05-00OR22725 at Oak Ridge National Laboratory.
[1] H.Leutwyler, these proceedings.

[2] I.Caprini, G.Colangelo and H.Leutwyler, Phys. Rev. Lett. 96, 132001 (2006) arXiv:hep-ph/0512364.

[3] A.Gal, these proceedings.

[4] A.Gal, arXiv:nucl-th/0607067

[5] J.A.Niskanen, these proceedings.

[6] H.Machner and J.Niskanen, arXiv:nucl-ex/0511027

[7] L.Tiator, these proceedings.

[8] L.Tiator and S.Kamalov, arXiv:nucl-th/0603012

[9] M.Soyeur, these proceedings.

[10] M.Soyeur and M.F.M.Lutz, arXiv:nucl-th/0607019

[11] C.Downum, T.Barnes, J.R.Stone and E.S.Swanson, Phys. Lett. B 638, 455 (2006) arXiv:nucl-th/0603020.

[12] M.M.Nagels, T.A.Rijken and J.J.deSwart, Phys. Rev. D 20, 1633 (1979).

[13] U.Mosel, these proceedings.

[14] K.Gallmeister, T.Leitner, S.Leupold, U.Mosel, P.Muehlich, L.Alvarez-Ruso and V.Shklyar, arXiv:nucl-th/0608025

[15] K.Seth, these proceedings.

[16] A.Krassnigg, these proceedings.
[17] A.Holl, A.Krassnigg, P.Maris, C.D.Roberts and S.V.Wright, Phys. Rev. C 71, 065204 (2005) arXiv:nucl-th/0503043.

[18] S.Kabana, these proceedings.

[19] S.Kabana, AIP Conf. Proc. 756, 195 (2005) arXiv:hep-ex/0503020.

[20] T.Barnes, AIP Conf. Proc. 814, 735 (2006) arXiv:hep-ph/0510365.

[21] M.Brooks, Young Frankenstein, Twentieth Century Fox (1974).

[22] R.L.Jaffe, Phys. Rev. Lett. 38, 195 (1977) [Erratum-ibid. 38, 617 (1977)].

[23] N.Isgur, Acta Physica Austriaca Suppl. XXVII, 177 (1985). Published in Schladming School 1985:0177 (QCD161:I8:1985), also in Los Alamos Wkshp. 1985:0619 (QCD161:W675:1985).

[24] T.Barnes, Acta Phys. Polon. B 31, 2745 (2000) arXiv:hep-ph/0008169.

[25] R.P.Feynman, Caltech commencement address (1974). 